\title{
MLKL-dependent epithelial-to-mesenchymal transition in nasopharyngeal carcinoma: a novel finding and avenues for future research
}

\author{
Tristan Tham
}

Department of Otolaryngology-Head and Neck Surgery, Zucker School of Medicine at Hofstra/Northwell, Lenox Hill Hospital, New York, NY, USA

Correspondence to: Tristan Tham, MD. Department of Otolaryngology-Head and Neck Surgery, Zucker School of Medicine at Hofstra/Northwell, 130 East $77^{\text {th }}$ Street, Lenox Hill Hospital, New York, NY 10075, USA. Email: tham@northwell.edu.

Provenance and Peer Review: This article was commissioned by the Editorial Office, Annals of Translational Medicine. The article did not undergo external peer review.

Comment on: Dong Y, Sun Y, Huang Y, et al. Depletion of MLKL inhibits invasion of radioresistant nasopharyngeal carcinoma cells by suppressing epithelial-mesenchymal transition. Ann Transl Med 2019;7:741.

Submitted Dec 23, 2019. Accepted for publication Jan 09, 2020.

doi: $10.21037 /$ atm.2020.01.83

View this article at: http://dx.doi.org/10.21037/atm.2020.01.83

\section{Introduction}

Nasopharyngeal carcinoma (NPC) is a cancer of the head and neck anatomical sub-site within the nasopharynx. The most common variety of NPC is the undifferentiated subtype, which is endemic to certain regions in Asia, particularly in southern China. Advances in treatment modalities over the preceding years, particularly in combined systemic treatment with intensity-modulated radiotherapy (IMRT) have improved survival significantly in this disease (1). Despite the good local control achieved in T1-2 disease, which averages around $80-90 \%$, distant control remains a significant problem. Previous studies have shown a disparity between local control and distant control of $10-20 \%$ (1). Treatment outcomes for metastatic NPC have been shown to be poor, with no effective systematic therapy to date for this group of patients (1). Therefore, research into mechanisms of distant metastases and spread are important goals in this disease entity.

One mechanism that a cancer may have a propensity to generate distant metastases is through epithelial-tomesenchymal transition (EMT), whereby cells lose their epithelial phenotype and gain a mesenchymal phenotype instead, which subsequently encourages invasiveness and distant spread. The authors of the current study investigated MLKL (Mixed Lineage Kinase Domain
Like Pseudokinase) which has recently been implicated in a contemporary paradigm, 'necroptosis' $(2,3)$. Briefly, necroptosis has been described as a fusion of the two concepts of 'apoptosis' and 'necrosis', whereby it is a form of programmed (unlike necrosis) caspase-independent cell death (unlike apoptosis) (3). Recent studies have shown that the necroptosis process contributes to tumor invasiveness and prognosis (3). However, the exact prognostic effects of necroptosis driven by MLKL are still uncertain. Several studies report that high expression of MLKL is associated with improved overall survival (OS) in cervical cancer $(2,4)$, and lower expression of MLKL is associated with poorer OS in ovarian and colorectal cancer $(5,6)$. The converse scenarios have also been reported, with the finding that phosphorylated (activated) MLKL leads to poorer prognosis in colon and esophageal cancers $(7,8)$. The current data on how MLKL affects prognosis in NPC is lacking, therefore although the results of the current study do not specifically study prognosis, their results suggest that MLKL could be one of the drivers of distant metastasis, and thus poorer prognosis in radioresistant NPC.

\section{Discussion}

In this study (9), the Dong et al. used knockdown via siRNA silencing of MLKL and knockout via CRISPR/Cas9 
models of investigation. Knockdown (siRNA silencing) of MLKL resulted in decrease in invasive capacity of CR cells but not for CNE-2 cells in vitro. Similar pattern was observed in the MLKL knockout experiment where CR cells had significant diminished invasive capacity compared to CNE-2 cells. In other words, in vitro models showed that radioresistant CR cells were more susceptible to gene knockdown and knockout of MLKL. The in vivo model showed no significant lung metastases of CR-MLKL KO cells, demonstrating the lack of invasive/metastatic capacity compared to the CR and CNE-2 models.

A further KEGG pathway analysis revealed that MLKL knockout results in decreased VIM (vimentin, associated with mesenchymal cells) expression and increased CDH1 (e-Cadherin, associated with epithelial cells). Thus, showing that MLKL could be a contributor to the EMT pathway. They additionally confirmed these findings using qRT-PCR, showing that CR cells had higher expression of mesenchymal markers and lower epithelial markers. Knockout of MLKL on CR cells showed a reversal with lower mesenchymal markers and higher epithelial markers. In summary, radio-resistant CR cells had a phenotype that was conducive to EMT and metastasis.

To my knowledge, MLKL-driven EMT in NPC has not been investigated to date. However, there are studies that have looked at necroptotic pathways (RIP1/RIP3/MLKL) and how they affect NPC proliferation. For example, a naphthoquinone compound that induces necroptosis through RIPK1/RIPK3/MLKL has been shown to inhibit NPC cell growth in vitro (10). That being said, these two lines of investigation should be viewed as distinct, as currently there is insufficient evidence to form a unified mechanism that explains both MLKL-driven EMT and how that interacts with the necroptotic pathway involving RIP1/RIP3/MLKL.

The authors illustrate a framework where EMT could theoretically be reversed with MLKL inhibition in NPC. However, more works needs to be performed to elucidate whether small-molecule inhibition of the MLKL protein would deliver similar results in the in vivo model. Further, the necroptosis effect of MLKL and receptor interacting protein kinase 1 and 3 (RIPK1 and RIPK3) are unknown in NPC, and should be a further avenue of research. Lastly, previous studies have shown that MLKL expression has been associated with improved tumor control by releasing damage-associated molecular patterns (DAMPs) to stimulate cross-priming of antitumoral CD8+ T-cells (3). Whether or not MLKL stimulates these intra-tumoral immune interactions in NPC remains to be investigated.

\section{Conclusions}

Dong et al. have illustrated that MLKL is a prospective driver candidate for EMT in NPC. Since distant control is poor within NPC, and treatment modalities for metastatic disease remain poor, research into EMT and metastatic mechanisms would be a key pathway to improving prognosis in NPC. Further work would be needed to better delineate the local and immune effects of MLKL inhibition/downregulation.

\section{Acknowledgments}

Funding: None.

\section{Footnote}

Conflicts of Interest: The author has no conflicts of interest to declare.

Ethical Statement: The author is accountable for all aspects of the work in ensuring that questions related to the accuracy or integrity of any part of the work are appropriately investigated and resolved.

Open Access Statement: This is an Open Access article distributed in accordance with the Creative Commons Attribution-NonCommercial-NoDerivs 4.0 International License (CC BY-NC-ND 4.0), which permits the noncommercial replication and distribution of the article with the strict proviso that no changes or edits are made and the original work is properly cited (including links to both the formal publication through the relevant DOI and the license). See: https://creativecommons.org/licenses/by-nc-nd/4.0/.

\section{References}

1. Lee AW, Ma BB, Ng WT, et al. Management of Nasopharyngeal Carcinoma: Current Practice and Future Perspective. J Clin Oncol 2015;33:3356-64.

2. Jiao D, Cai Z, Choksi S, et al. Necroptosis of tumor cells leads to tumor necrosis and promotes tumor metastasis. Cell Res 2018;28:868-70.

3. Qin X, Ma D, Tan YX, et al. The role of necroptosis in cancer: A double-edged sword? Biochim Biophys Acta Rev 
Cancer 2019;1871:259-66.

4. Ruan J, Mei L, Zhu Q, et al. Mixed lineage kinase domain-like protein is a prognostic biomarker for cervical squamous cell cancer. Int J Clin Exp Pathol 2015;8:15035-8.

5. He L, Peng K, Liu Y, et al. Low expression of mixed lineage kinase domain-like protein is associated with poor prognosis in ovarian cancer patients. Onco Targets Ther 2013;6:1539-43.

6. Li X, Guo J, Ding AP, et al. Association of Mixed Lineage Kinase Domain-Like Protein Expression With Prognosis in Patients With Colon Cancer. Technol Cancer Res Treat 2017;16:428-34.

7. Bozec D, Iuga AC, Roda G, et al. Critical function of the

Cite this article as: Tham T. MLKL-dependent epithelialto-mesenchymal transition in nasopharyngeal carcinoma: a novel finding and avenues for future research. Ann Transl Med 2020;8(5):153. doi: 10.21037/atm.2020.01.83 necroptosis adaptor RIPK3 in protecting from intestinal tumorigenesis. Oncotarget 2016;7:46384-400.

8. Liu X, Zhou M, Mei L, et al. Key roles of necroptotic factors in promoting tumor growth. Oncotarget 2016;7:22219-33.

9. Dong Y, Sun Y, Huang Y, et al. Depletion of MLKL inhibits invasion of radioresistant nasopharyngeal carcinoma cells by suppressing epithelial-mesenchymal transition. Ann Transl Med 2019;7:741.

10. Liu T, Sun X, Cao Z. Shikonin-induced necroptosis in nasopharyngeal carcinoma cells via ROS overproduction and upregulation of RIPK1/RIPK3/MLKL expression. Onco Targets Ther 2019;12:2605-14. 